\title{
Absorption Imaging of Ultracold Neutral Plasmas
}

\author{
C. E. Simien, Y. C. Chen, P. Gupta, S. Laha, Y. N. Martinez, P. G. Mickelson, S. B. Nagel, and T. C. Killian
}

\begin{abstract}
We report optical absorption imaging of ultracold neutral plasmas. Imaging allows direct observation of the ion density profile and expansion of the plasma. The frequency dependence of the plasma's optical depth gives the ion absorption spectrum, which is broadened by the ion motion. We use the spectral width to monitor ion equilibration in the first $250 \mathrm{~ns}$ after plasma formation. On a microsecond time scale, we observe the radial acceleration of ions resulting from pressure exerted by the trapped electron gas.
\end{abstract}

Index Terms-Image analysis, plasma measurements, plasmas, spectral analysis.

$\mathbf{U}$ LTRACOLD neutral plasmas are systems in which particle temperatures can be $1 \mathrm{~K}$ or lower. Fundamental interest in these systems stems from a range of phenomena that occur in the ultracold regime. For instance, it may be possible to form strongly coupled systems in which the electrical interaction energy between the charged particles exceeds the average kinetic energy. This reverses the traditional energy hierarchy that underlies our normal understanding of plasmas based on concepts such as Debye screening and hydrodynamics.

To produce ultracold plasmas, laser-cooled atoms [1], [2] are ionized with photons from a laser whose wavelength is tuned barely above the ionization threshold. Due to the small electron mass, the initial electron kinetic energy $E_{e}$ approximately equals the difference between the photon energy and the ionization potential, which can be as low as a $100 \mathrm{mK}$. The initial kinetic energy of the ions is in the millikelvin range. The resulting nonequilibrium plasma evolves rapidly, and many of its characteristics remain to be studied. Experiments [3]-[5] conducted so far reveal surprising dynamics and recombination behavior that show us we are stretching the bounds of plasma physics. Many theory papers have addressed the plasma behavior [6]-[10].

We report results with a new diagnostic tool to study ultracold neutral plasmas: absorption imaging of a strontium plasma using the $\mathrm{Sr}^{+} 2 S_{1 / 2} \rightarrow 2 P_{1 / 2}$ transition at $422 \mathrm{~nm}$ [11]. This technique provides in situ, nondestructive measurements and offers excellent spatial, temporal, and spectral resolution. The absorption imaging reported here is particularly well adapted for small, cold, and relatively dilute plasmas that evolve very quickly.

A collimated laser beam, tuned near resonance with the principal transition in the ions, illuminates the plasma and falls on an image intensified charge-coupled device (CCD) camera. Following Beer's law, the optical depth (OD) is defined in terms of

Manuscript received July 1, 2004; revised January 3, 2005.

The authors are with the Department of Physics and Astronomy, Rice University, Houston TX 77251 USA (e-mail: simien@ rice.edu).

Digital Object Identifier 10.1109/TPS.2005.845342 the image intensity without $\left(I_{\text {background }}\right)$ and $\left(I_{\text {plasma }}\right)$ with the plasma present

$$
\begin{aligned}
\mathrm{OD}(x, y) & =\operatorname{In}\left(I_{\text {background }}(x, y) / I_{\text {image }}(x, y)\right) \\
& =\int_{-\infty}^{\infty} d z n_{i}(x, y, z) \alpha\left[\nu, T_{i}(r)\right]
\end{aligned}
$$

where $n_{i}(x, y, z)$ is the ion density, and $\alpha\left[\nu, T_{i}(r)\right]$ is the ion absorption cross section at the image beam frequency, $\nu$. The absorption cross section is a function of temperature due to Doppler broadening, and since we expect the temperature to vary with density, we allow $\alpha$ to vary with position.

Fig. 1 shows a typical absorption image. The intensity of the probe beam is much less than the saturation intensity of the transition. The spatial resolution of typical images is 65 limited by pixel averaging performed to improve the signal-to-noise ratio of the images.

To obtain the absorption spectrum, we plot the integral of the optical depth over $\mathrm{x}$ and $\mathrm{y}$ as a function of the imaging laser frequency. For longer delay times $\left(t_{\text {delay }}\right)$ between formation of the plasma and camera exposure, the spectral lines become significantly broader than the natural linewidth of the transition. The dominant contribution to the linewidth beyond the natural linewidth is Doppler broadening, which makes the absorption spectrum a very accurate probe of the ion velocity distribution. From a fit of each spectrum to a Voigt profile using a Lorenztian width equal to the sum of the natural width and the laser linewidth, we extract the root mean square (rms) Doppler broadening, $\sigma_{D}=\sqrt{\left(k_{B} T_{i, \text { eff }} / m_{i}\right)} /(\gamma)$, where $m_{i}$ is the ion mass, and $\lambda$ is the wavelength of the transition. This provides the effective ion temperature $T_{i \text {,eff }}$ as a function of time. Various effects contribute to the ion kinetic energy and thus to $T_{i \text {,eff }}$.

$T_{i \text {,eff }}$ increases rapidly for $t_{\text {delay }}<200 \mathrm{~ns}$. Two pieces of information imply that this is thermalization of ions with themselves after creation in a spatially disordered state [6]: 1) the time scale is on the order in which ions respond to perturbations from their equilibrium spatial distribution, which is on the order of the inverse plasma frequency of ions, $\tau_{i}=\omega_{\mathrm{pi}}^{-1}=$ $\sqrt{m_{i} \epsilon_{0} / n_{0 i} e^{2}} \approx 100 \mathrm{~ns}$, where and are the electric charge and initial peak ion density; 2) the energy is also on the order of $e^{2} / 4 \pi \epsilon_{0} a k_{b}$ the amount of potential energy inherent in the initial disorder of the ions. Here, $a=\left(4 \pi n_{0 e} / 3\right)^{-1 / 3}$ is the Wigner-Seitz radius, and is the initial peak electron density.

For $t_{\text {delay }}>200 \mathrm{~ns}, T_{i, \text { eff }}$ continues to increase, but at a slower rate. The slow increase results from an outward radial acceleration of the ions caused by pressure exerted by the gas of trapped electrons [9], [10]. This acceleration will lead to the expansion of the plasma on a $10-\mu$ s time scale.

In conclusion, absorption imaging of ultracold neutral strontium plasma is a powerful diagnostic technique. We use this 


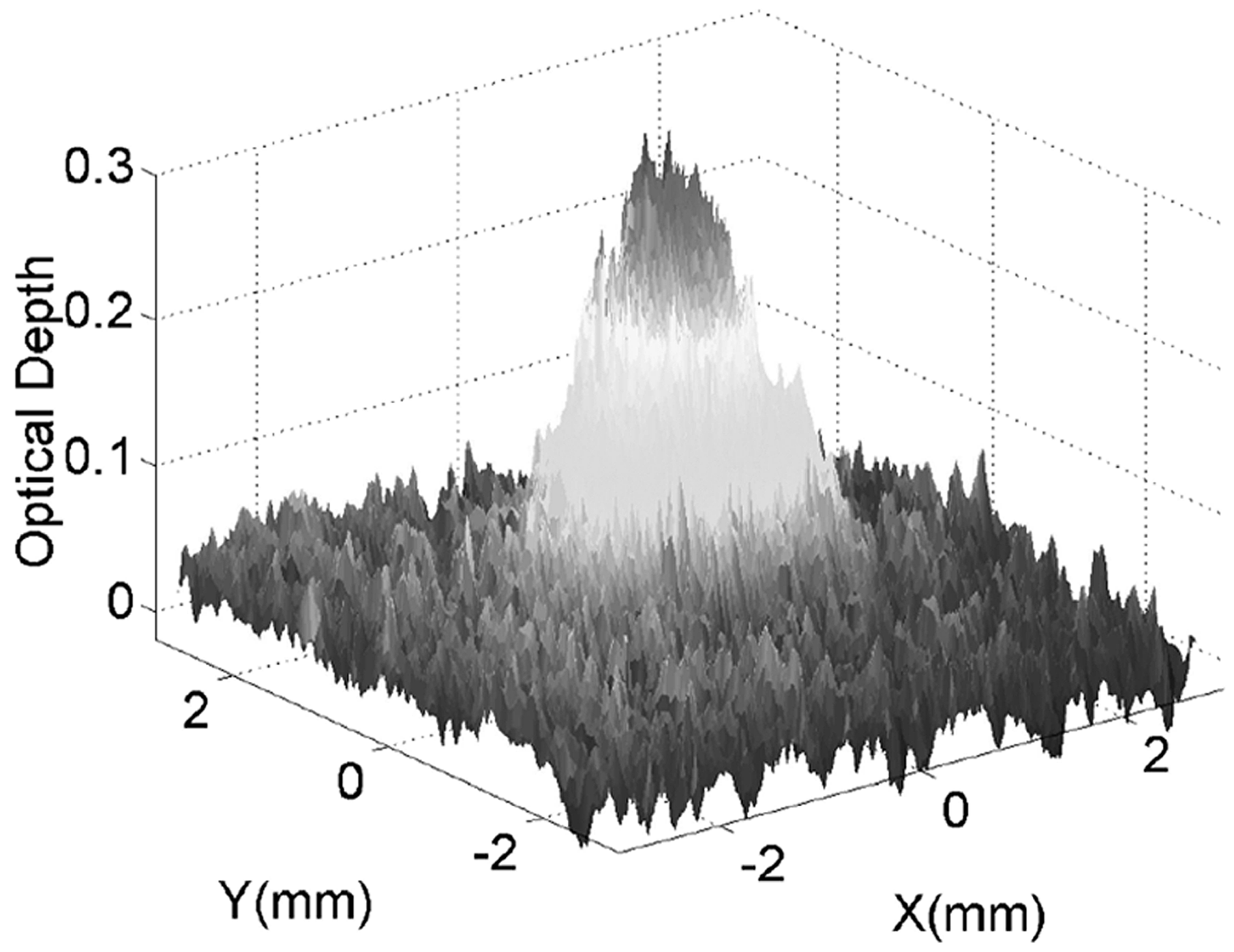

Fig. 1. Optical depth of an ultracold neutral plasma. Delay between the formation of the plasma and image exposure is $85 \mathrm{~ns}$, and the initial peak ion density is $n_{0 i}=2 \times 10^{10} \mathrm{~cm}-3$. Plasma contains $7 \times 10^{7}$ ions. Spatial resolution is about $65 \mu \mathrm{m}$, limited by pixel averaging performed to improve the signal-to-noise ratio.

technique to study the ion spectrum at various delay times. The spectrum reveals rapid thermalization of the ions on a submicrosecond time scale as predicted by theory [6], and the acceleration of ions due to electron pressure. In addition, this probe has the potential to study a host of phenomena such as ion collective modes, shock waves, recombination, and particle-particle spatial correlations.

\section{REFERENCES}

[1] H. J. Metcalf and P. van der Straten, Laser Cooling and Trapping. New York: Springer-Verlag, 1999.

[2] S. B. Nagel, C. E. Simien, S. Laha, P. Gupta, V. S. Ashoka, and T. C. Killian, "Magnetic trapping of metastable ${ }^{3} P_{2}$ atomic strontium," Phys. Rev. A., Gen. Phys., vol. 67, pp. 011 401-1-011 401-4, 2003.

[3] T. C. Killian, S. Kulin, S. D. Bergerson, L. A. Orozco, C. Orzel, and S. L. Rolston, "Creation of an ultracold neutral plasma," Phys. Rev. Lett., vol. 83, pp. 4776-4779, 1999.
[4] S. Kulin, T. C. Killian, S. D. Bergeson, and S. L. Rolston, "Plasma oscillations and expansion of an ultracold neutral plasma," Phys. Rev. Lett., vol. 85, pp. 318-321, 2000.

[5] T. C. Killian, M. J. Lim, S. Kulin, R. Dumke, S. D. Bergeson, and S. L. Rolston, "Formation of rydberg atoms in an expanding ultracold neutral plasma," Phys. Rev. Lett., vol. 86, pp. 3759-3762, 2001.

[6] M. S. Murillo, "Using fermi statistics to create strongly coupled ion plasmas in atom traps," Phys. Rev. Lett., vol. 87, pp. 115 003-1-115003-4, 2001.

[7] S. G. Kluzmin and T. M. O'Neil, "Numerical simulation of ultracold plasmas: How rapid intrinsic heating limits the development of correlation," Phys. Plasmas, vol. 9, pp. 0605 003-0605 004, 2002.

[8] S. Mazevet, L. A. Collins, and J. D. Kress, "Evolution of ultracold neutral plasmas," Phys. Rev. Lett., vol. 88, pp. 055 001-1-055 001-4, 2002.

[9] F. Robicheaux and J. D. Hanson, "Simulation of the expansion of an ultracold neutral plasma," Phys. Plasmas, vol. 10, pp. $055002-1-055002-4,2003$.

[10] T. Pohl, T. Pattard, and J. M. Rost, "Coulomb crystallization in expanding laser-cooled neutral plasmas," Phys. Rev. Lett., vol. 92, pp. 155 003-1-155 003-4, 2004.

[11] C. E. Simien, Y. C. Chen, P. Gupta, S. Laha, Y. N. Martinez, P. G. Mickelson, S. B. Nagel, and T. C. Killian, "Using absorption imaging to study ion dynamics in an ultracold neutral plasma," Phys. Rev. Lett., vol. 92, pp. $143001-1-143001-4,2004$. 